\title{
DNAJC25 is downregulated in hepatocellular carcinoma and is a novel tumor suppressor gene
}

\author{
TINGTING LIU, WEI JIANG, DINGDING HAN and LONG YU \\ State Key Laboratory of Genetic Engineering, Institute of Genetics, School of Life Sciences, \\ Fudan University, Shanghai 200433, P.R. China
}

Received May 8, 2012; Accepted July 26, 2012

DOI: $10.3892 / \mathrm{ol} .2012 .903$

\begin{abstract}
HSP40, also known as DnaJ, is one of the subfamilies of the heat shock protein family. DnaJ/Hsp40 proteins act as co-chaperones by binding to the chaperone Hsp70 through their J domain and stimulating ATP hydrolysis to aid protein translation, folding, unfolding, translocation and degradation. They are implicated in various human diseases, including neurodegenerative disorders and cancer. In the present study, we cloned and identified a new gene, DnaJ (HSP40) homolog, subfamily C, member 25 (DNAJC25), which is localized to the cytoplasm. Real-time PCR revealed that the expression of DNAJC25 is particularly high in the liver and is downregulated in hepatocellular carcinoma (HCC) compared with adjacent normal tissues. The overexpression of DNAJC25 led to an inhibition of colony growth both in quantity and size. Flow cytometry analysis indicated that DNAJC25 also significantly increased cell apoptosis. Our data, therefore, indicate that DNAJC25 plays an important role in hepatocellular carcinogenesis, and should be further studied as a potential tumor suppressor candidate.
\end{abstract}

\section{Introduction}

Hepatocellular carcinoma (HCC) is one of the most common malignant tumors and ranks third as the leading cause of cancer-related mortalities worldwide (1). Due to its late diagnosis, ineffectiveness of conventional treatments and poor prognosis, the mortality rate for $\mathrm{HCC}$ is extremely high $(2,3)$. Therefore, more effective treatments and more accurate and reliable diagnostic markers are needed to provide earlier detection and better therapeutic strategies.

Correspondence to: Professor Long Yu, State Key Laboratory of Genetic Engineering, Institute of Genetics, School of Life Sciences, Fudan University, 220 Handan Road, Shanghai 200433, P.R. China E-mail: longyu@fudan.edu.cn

Key words: heat shock protein, downregulated, hepatocellular carcinoma, apoptosis, tumor suppressor
Heat shock proteins (HSPs) are a group of proteins whose expression is initially induced by heat. Further study revealed the same response in HSPs exposed to other environmental and metabolic stimulations, including hypoxia, hyperoxia, anoxia, UV exposure and mechanical injury. In addition, HSPs have also been found in cells under normal conditions $(4,5)$. Previous studies have revealed that HSPs are ubiquitous in both prokaryotic and eukaryotic cells. They have dual functions: the intracellular HSPs, which are known as 'molecular chaperones', have cytoprotective and antiapoptotic functions, such as ensuring the folding of proteins into the correct tertiary structure, transporting proteins across the membranes and incorporating polypeptides into intracellular membranes; the extracellular HSPs have certain immunogenic functions, through the chaperoning of antigenic peptides $(6,7)$.

Since the molecular chaperone functions of HSPs have been shown to be important in regulating cellular homeostasis and promoting cell survival, the proteins have been reported to participate in the progression of numerous types of diseases, including autoimmune diseases and neoplastic processes (8). As the overexpression of HSP27, 70 and 90 has been detected in various types of carcinoma compared with corresponding normal tissues, the correlation between HSPs and cancer has attracted attention (9). Following studies have not only focused on the correlation between HSP expression profiles, prognosis and clinical indicators, including disease classification, invasion, metastasis and therapeutic resistance, but have also described the function of HSPs in carcinogenesis $(10,11)$.

In the present study, we investigated a novel gene called DNAJC25, which belongs to the HSP40 subfamily C. We cloned the gene and explored the subcellular localization of the protein in the cytoplasm. We found that DNAJC25 had a markedly high expression level in liver tissues and was significantly downregulated in liver cancer tissues compared with the adjacent normal tissues. The overexpression of DNAJC25 reduced colony formation and limited the colony quantity and size. Flow cytometry analysis indicated that DNAJC25 also significantly increased cell apoptosis. The present study describes a new potential tumor suppressor and furthers the understanding of the function of HSPs in cancer progression. 


\section{Materials and methods}

Tumor specimens. Fresh surgical specimens of HCC, including tumor tissues and the neighboring pathologically nontumorous liver tissues, were obtained from liver cancer patients at Zhongshan Hospital (Shanghai, China). All the samples were immediately frozen in liquid nitrogen after surgery and stored at $-80^{\circ} \mathrm{C}$ before further analysis.

Cloning and sequencing of DNAJC25. Two primers (forward, 5'-TGAGTGCTGCAGAATCGCTGG-3'; and reverse, 5'-AAG GTTTGGCATAGTAGCATTCCATC-3') were designed to amplify DNAJC25 from a human testis cDNA library. PCR was performed using a PCR kit (SH Energy, Driffield, UK) under conditions of $95^{\circ} \mathrm{C}$ for $5 \mathrm{~min}, 40$ cycles of $95^{\circ} \mathrm{C}$ for $30 \mathrm{sec}, 64^{\circ} \mathrm{C}$ for $30 \mathrm{sec}$ and $72^{\circ} \mathrm{C}$ for $1 \mathrm{~min} 20 \mathrm{sec}$, followed by 1 cycle of $72^{\circ} \mathrm{C}$ for $10 \mathrm{~min}$. The product was then separated by DNA electrophoresis in $1 \%(\mathrm{w} / \mathrm{v})$ agarose gel and inserted into pMD18-T vector (Takara Bio, Inc., Otsu, Japan) for sequencing. The cDNA encoding DNAJC25 from the pMD18-T vector was then subcloned into pCMV-Myc (Clontech Laboratories, Inc., Mounatin View, CA, USA), pcDNA3.1A(-) and pEGFP-N1 for the evaluation of eukaryotic expression and further analysis.

RT-PCR analysis. Human multiple tissue cDNA (MTC) panels (Clontech Laboratories, Inc.), including heart, brain, placenta, lung, liver, skeletal muscle, kidney, pancreas, spleen, thymus, prostate, testis, ovary, small intestine and colon, served as templates to study the distribution of human DNAJC25 mRNA. Primer pairs designed at the boundary between exons 1 and 2 and exons 3 and 4 (forward, 5'-AGA CACTCAAGGATGAAGAAACAC-3'; reverse, 5'-TTGCTT GTAGACCTCATAATTCTCC-3') were used for RT-PCR under conditions of $95^{\circ} \mathrm{C}$ for $5 \mathrm{~min}, 38$ cycles of $95^{\circ} \mathrm{C}$ for $30 \mathrm{sec}, 63^{\circ} \mathrm{C}$ for $30 \mathrm{sec}$ and $72^{\circ} \mathrm{C}$ for $40 \mathrm{sec}$, followed by 1 cycle of $72^{\circ} \mathrm{C}$ for $10 \mathrm{~min}$. 5'-ATGAGTATGCCTGCCGTG TGAAC-3' and 5'-TGTGGAGCAACCTGCTCAGATAC-3' were used to amplify the $\beta 2-\mathrm{MG}$ gene. The products were then separated by DNA electrophoresis in $2 \%$ (w/v) agarose gel.

Cell culture and transfection. HEK 293, SMMC-7721, Hep3B and HeLa cells were cultured in Dulbecco's modified Eagle's medium (Gibco, Invitrogen Life Technologies, Carlsbad, CA, USA) supplemented with $10 \%$ fetal bovine serum (Invitrogen Life Technologies) at $37^{\circ} \mathrm{C}$ in a $5 \% \mathrm{CO}_{2}$ humidified atmosphere. HEK 293 cells $\left(1.2 \times 10^{5}\right)$ were seeded in a 6 -well plate, 3.0x10 5 SMC-7721 and Hep3B cells were seeded in a 6 -well plate and $1.5 \times 10^{5} \mathrm{HeLa}$ cells were seeded on coverslips in a 6-well plate. After overnight growth, the cells were $60 \%$ confluent and were transfected with the plasmids using Lipofectamine Reagent (Invitrogen Life Technologies) in the non-serum medium. After $4 \mathrm{~h}$ of incubation, the medium was replaced with fresh complete medium, and cells were cultured for an additional $36 \mathrm{~h}$ before collection.

Quantitative real-time PCR. RNA was extracted with TRIzol (Invitrogen Life Technologies) and reverse transcribed to cDNA with a reverse transcription kit (Invitrogen Life
Technologies) according to the manufacturer's instructions. Quantitative real-time PCR was performed using the SYBR Green Supermix kit (Takara Bio, Inc.) with the Light Cycler 480 (Roche Diagnostics, Mannheim, Germnay). For both DNAJC25 and the house keeping gene $\beta 2-\mathrm{MG}$, cycle parameters were $95^{\circ} \mathrm{C}$ for $1 \mathrm{~min}$ hot start, followed by 45 cycles of $95^{\circ} \mathrm{C}$ for $10 \mathrm{sec}, 63^{\circ} \mathrm{C}$ for $10 \mathrm{sec}$ and $72^{\circ} \mathrm{C}$ for $40 \mathrm{sec}$. The primers for DNAJC25 were: forward, 5'-AGACACTCAAGG ATGAAGAAACAC-3'; reverse, 5'-TTGCTTGTAGACCTC ATAATTCTCC-3'. The primers for $\beta 2-\mathrm{MG}$ were: forward, 5'-ATGAGTATGCCTGCCGTGTGAAC-3'; reverse, 5'-TGT GGAGCAACCTGCTCAGATAC-3'. Quantified transcripts of the $\beta 2-M G$ gene were used as endogenous mRNA controls. All experiments were performed thrice for each data point.

Western blot analysis. Protein samples separated by SDS-PAGE were electrotransferred onto a nitrocellulose membrane. The membrane was blocked at room temperature for $1 \mathrm{~h}$ with TBS containing 5\% (w/v) skimmed milk. The membrane was then incubated overnight at $4^{\circ} \mathrm{C}$ with mouse anti-Myc monoclonal antibody (dilution, 1:1,000; Sigma, St. Louis, MO, USA), washed three times with a mixture of TBS with 0.1\% Tween-20 (Sigma; TBS-T) and incubated with HRP-conjugated goat anti-mouse antibody (dilution, 1:5,000; Santa Cruz Biotechnology Inc., Santa Cruz, CA, USA) at room temperature for $1 \mathrm{~h}$. The membrane was then washed again with TBS-T and developed using the ECL system (Santa Cruz Biotechnology Inc.).

Immunofluorescence microscopy. SMMC-7721 and HeLa cells were plated on coverslips and transfected with lipofectamine (Invitrogen Life Technologies). After $36 \mathrm{~h}$, the cells were washed twice with PBS ( $\mathrm{pH} 7.4$ ) and fixed in $4 \%$ paraformaldehyde for $10 \mathrm{~min}$ at room temperature. Then cells were resolved by $0.1 \%$ (v/v) Triton X-100 for $5 \mathrm{~min}$, washed again and stained with DAPI for $2 \mathrm{~min}$ at room temperature in the dark. Images were viewed using LSM 710 Laser Scanning Microscopy (Carl Zeiss, Cambridge, UK).

Colony formation assay. At $24 \mathrm{~h}$ post-transfection with pcDNA3.1A(-)-DNAJC25 and pcDNA3.1A(-) vector, Hep3B and SMMC-7721 were portioned into new 6-well plates at densities of $2.5 \times 10^{4}$ and $1 \times 10^{5}$ cells/well, respectively, in triplicate. G418 (500 $\mu \mathrm{g} / \mathrm{ml}$; Invitrogen Life Technologies) was added to the medium $24 \mathrm{~h}$ later. The colonies were identified by crystal violet staining after $\sim 10-14$ days of culture. The results are representative of at least three independent experiments.

Flow cytometry analysis. After transfection for $36 \mathrm{~h}$, cells were harvested and incubated with RNAase $(100 \mu \mathrm{g} / \mathrm{ml})$ and propidium iodide $(50 \mu \mathrm{g} / \mathrm{ml})$ for $5 \mathrm{~min}$ at room temperature. The degree of apoptosis was indicated by the percentage of cells in the sub-G1 fraction, using FACSCalibur (BD Biosciences, Franklin Lakes, NJ, USA).

Statistical analysis. A two-tailed Student's t-test was used to evaluate group-level differences in our study. $\mathrm{P}<0.05$ was considered to indicate a difference and $\mathrm{P}<0.01$ was considered to indicate a statistically significant difference. 


\section{Results}

Molecular cloning and identification of human DNAJC25. Through PCR from the human testis cDNA library with the forward and reverse primers, an amplicon was obtained and inserted into the pMD18-T vector (Fig. 1A). After sequencing, we found that the cDNA of the amplicon was $1163 \mathrm{bp}$ in length. Online BLAST research (http://www.ncbi.nlm.nih.gov/blast) revealed that the amplicon sequence was identical to the mRNA sequence of DNAJC25 (GenBank ID, GI:118498346) and that they have the same ORF. Fig. 1B shows the evolutionary relationship of DNAJC25 with its homologous genes in other species. Bioinformatic analysis revealed that DNAJC25 was located at chromosome 9 (Fig. 1C) and that DNAJC25 cDNA encodes a putative 360 -amino acid protein with a calculated molecular mass of $42.4 \mathrm{kDa}$.

Subcellular localization of DNAJC25 in eukaryotic cells. In order to study the subcellular localization of DNAJC25, we cloned DNAJC25 into the pEGFP-N1 vector and transfected the construct into SMMC-7721 and HeLa cells. The pEGFP-N1 vector was used as a control. The cells were fixed and observed under fluorescence microscopy $36 \mathrm{~h}$ post-transfection. The results showed that DNAJC25 was localized to the cytoplasm in SMMC-7721 and HeLa cells (Fig. 2).

DNAJC25 had a markedly high expression in liver tissue. RT-PCR was performed to detect the distribution of DNAJC25 in 15 human tissues with the primers located at the boundary between exons 1 and 2 and exons 3 and 4. The results showed that DNAJC25 had the highest expression level in liver tissue and trace levels in the thymus, prostate, testis, ovary, small intestine and colon. No amplification product was visualized in the heart, brain, placenta, lung, skeletal muscle, kidney, pancreas or spleen (Fig. 3A).

DNAJC25 was downregulated in HCC. Since the expression level of DNAJC25 was extremely high in liver tissues, the mRNA level of DNAJC25 was further evaluated in 87 pairs of HCC specimens and their corresponding neighboring nontumorous specimens by quantitative real-time PCR. Quantified transcripts of the $\beta 2-\mathrm{MG}$ gene were used as endogenous mRNA controls. Fig. 3B shows the $\log _{2}$-transformed fold changes of DNAJC25 mRNA expression as a ratio of tumor/nontumor levels. DNAJC25 was downregulated in $50 \mathrm{HCC}$ specimens compared with adjacent normal liver tissues $(57.5 \%$; $>2$-fold decrease). The DNAJC25 transcripts were normally expressed in 26 tumours $(29.9 \%)$ and overexpressed in 11 tumours (12.6\%; $>2$-fold increase; $\mathrm{P}<0.001)$.

Colony formation assay. Since DNAJC25 was downregulated in HCC, we aimed to determine whether DNAJC25 was able to inhibit tumor growth. We performed a colony formation assay with HCC cells overexpressing DNAJC25. As shown in Fig. 4A, the overexpression of DNAJC25 markedly reduced the number of surviving colonies of Hep3B and SMMC-7721 cells. The mean reduction in colony formation was $74.67 \%$ for Hep3B $(\mathrm{P}<0.001)$ and $79.00 \%$ for SMMC-7721 cells $(\mathrm{P}<0.05)$ from three independent experiments (Fig. 4B). Colonies which formed in the group transfected with pcDNA3.1A(-)-DNAJC25
A

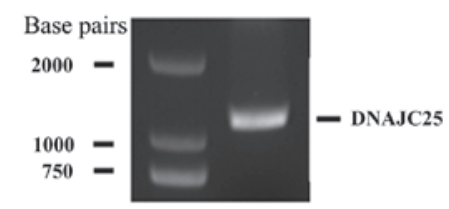

B

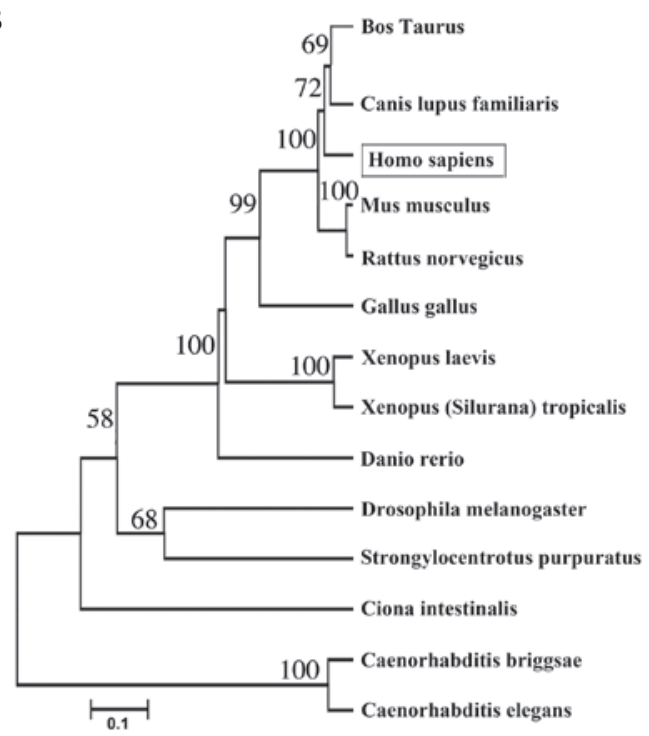

C

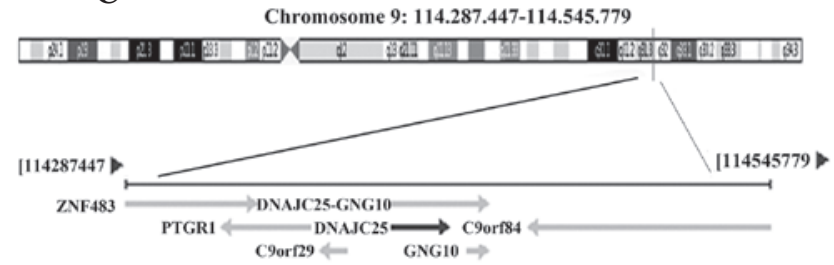

Figure 1. Molecular cloning and identification of DNAJC25. (A) Identification of DNAJC25 with PCR. Two primers were designed to amplify DNAJC25 from a human testis cDNA library and a 1163-bp amplicon was obtained. (B) The evolutionary relationship of DNAJC25 with its homologous genes in other species. The evolutionary tree was constructed by the molecular evolutionarygeneticsanalysis software MEGA5.05, using Neighbor-Joining method. (C) The location of DNAJC25 on chromosome 9. The information was acquired from the online databases GenBank and Ensembl.

were smaller in size than those formed in the group transfected with the pcDNA3.1A(-) vector. Our results suggest a suppressive role of DNAJC25 in cell survival or proliferation.

Overexpression of DNAJC25 induced apoptotic cell death. In order to study the role of DNAJC25 in cell proliferation, survival and cell cycle blocking, Hep3B and HEK 293 cells were transfected with DNAJC25 and analyzed by flow cytometry. Compared with the control group, Hep3B and HEK 293 cells transfected with DNAJC25 showed a marked sub-G1 peak, indicating a significantly increased apoptotic cell population. The sub-G1 ratio of the Hep3B cells transfected with pCMV-Myc-DNAJC25 was $18.80 \%$, while that of the control group was $13.23 \%(\mathrm{P}<0.001$; Fig. 5A). The sub-G1 ratio of the HEK 293 cells transfected with pCMV-Myc-DNAJC25 was $16.41 \%$ and that of the control group was $3.92 \%(\mathrm{P}<0.05$; Fig. 5B). However, there was no marked difference between cells transfected with pCMV-Myc-DNAJC25 and the control group in cell cycle progression. Our results indicate that DNAJC25 was able to induce apoptosis, but not cycle arrest. 


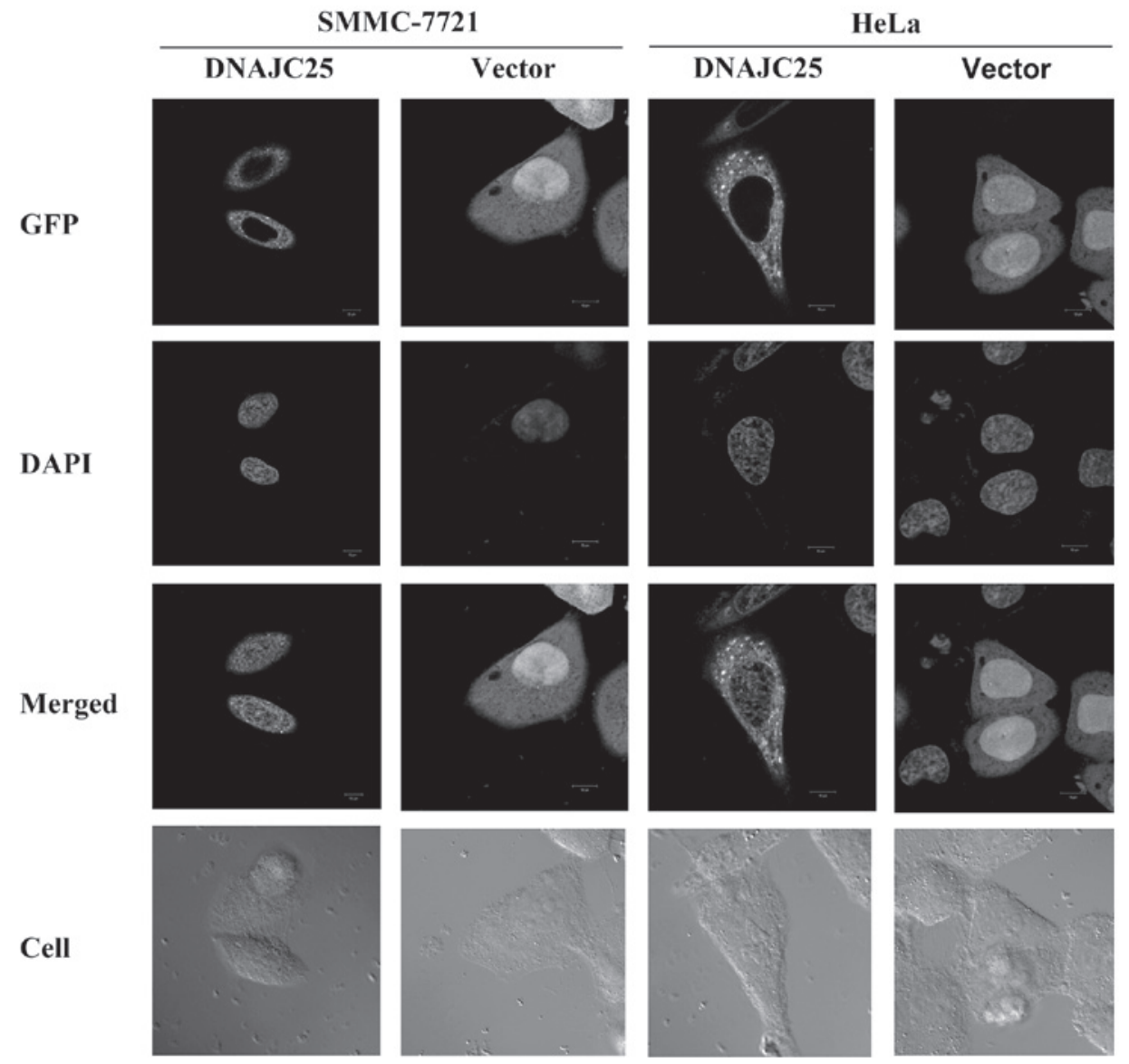

Figure 2. Subcellular localization of DNAJC25. DNAJC25 is located in the cytoplasm. SMMC-7721 and HeLa cells were transfected with pEGFP-N1-DNAJC25 construct (empty vector pEGFP-N1 was used as control). Cells were fixed by $4 \%$ paraformaldehyde and stained with DAPI for nuclei $36 \mathrm{~h}$ after transfection.

$\mathbf{A}$

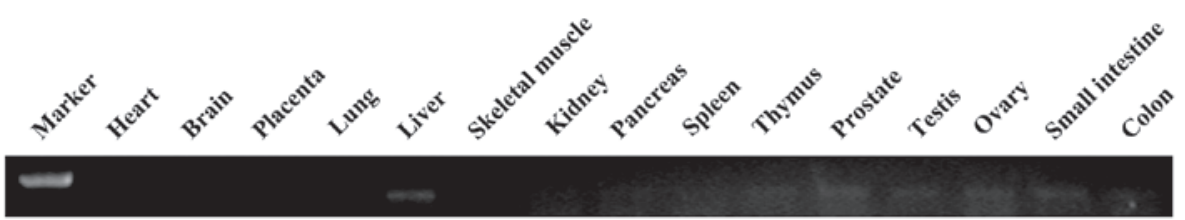

B2-MG

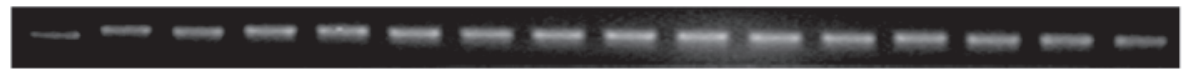

B

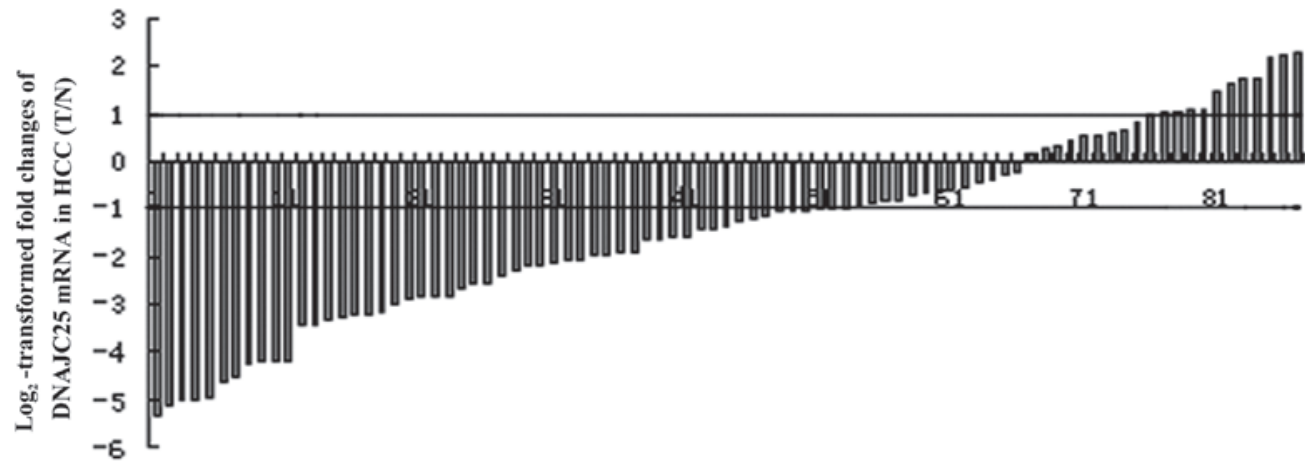

Figure 3. Expression profile of DNAJC25. (A) Distribution of DNAJC25 in 15 human tissues. DNAJC25 has the highest expression level in the liver and has trace levels in the thymus, prostate, testis, ovary, small intestine and colon, which were markedly lower than that in the liver. No amplification product was visualized from other tissues. As a control, the human $32-\mathrm{MG}$ gene was also amplified under the same conditions. (B) Downregulation of DNAJC25 expression in HCC specimens. DNAJC25 mRNA expression levels were analyzed in 87 paired HCC specimens with their corresponding neighboring nontumorous specimens (Nos. 1-87) by quantitative real-time PCR. $\beta 2-\mathrm{MG}$ was used as internal reference. Values are expressed as $\log _{2}$-transformed relative fold decrease or increase in the mRNA expression with respect to the adjacent nontumorous tissue. A positive $\log _{2}$-transformed fold change indicates increased expression levels in tumor specimens compared with the neighboring nontumorous specimens, whereas a negative value indicates relatively decreased levels. A 2-fold change threshold was set for identifying significant changes. HCC, hepatocellular carcinoma; T/N, tumor/nontumor tissue. 
A
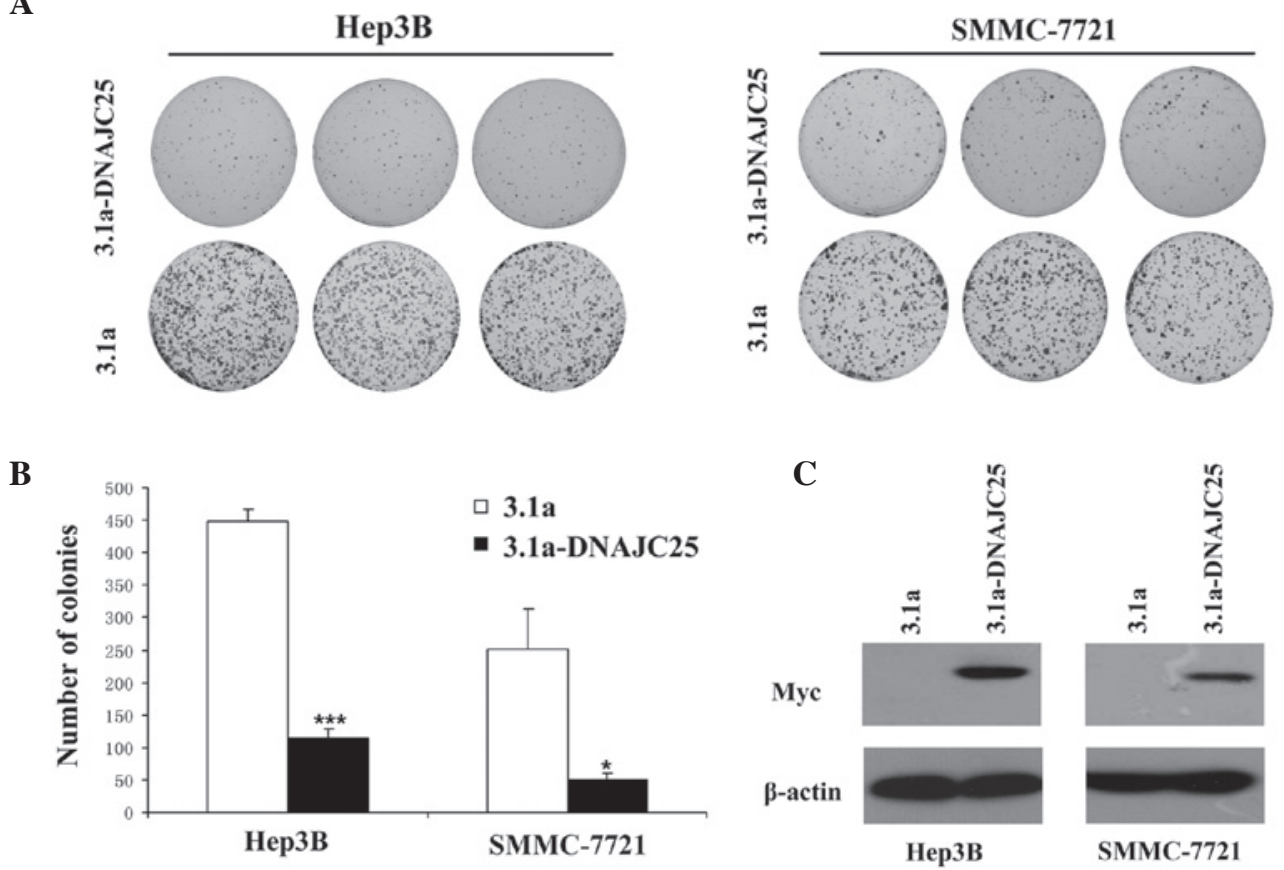

Figure 4. Ectopic expression of DNAJC25 reduced the growth characteristics of the HCC cell lines Hep3B and SMMC-7721. Cells were transiently transfected with pcDNA3.1A(-)-DNAJC25 or pcDNA3.1A(-) vector (control), split and cultured in the presence of G418. Colonies were identified by crystal violet staining after 10-14 days of culture. (A) Representative images of colony formation assay in Hep3B and SMMC-7721. (B) Number of colonies growing on plastic dishes of Hep3B and SMMC-7721 cell lines was counted. Columns, mean ( $\mathrm{n}=3)$; bars, SD. ${ }^{*} \mathrm{P}<0.05 ;{ }^{* * * *} \mathrm{P}<0.001$ compared with control. (C) Cellular extracts derived from cell tranfectants were analyzed by western blotting using the indicated antibodies.

A

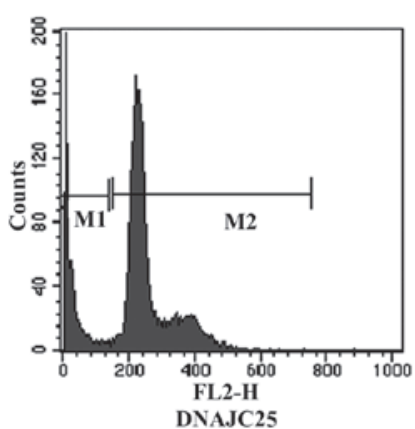

B

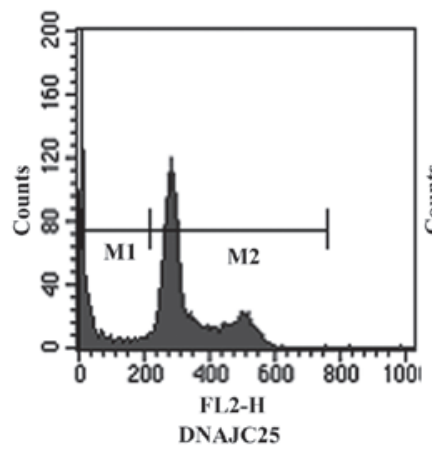

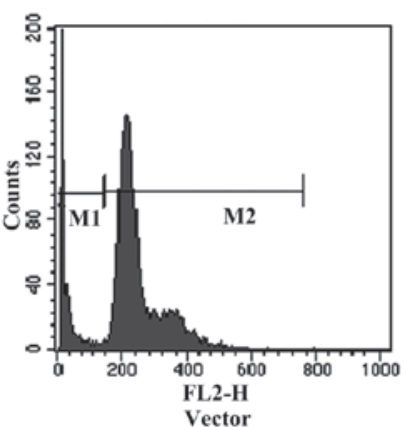
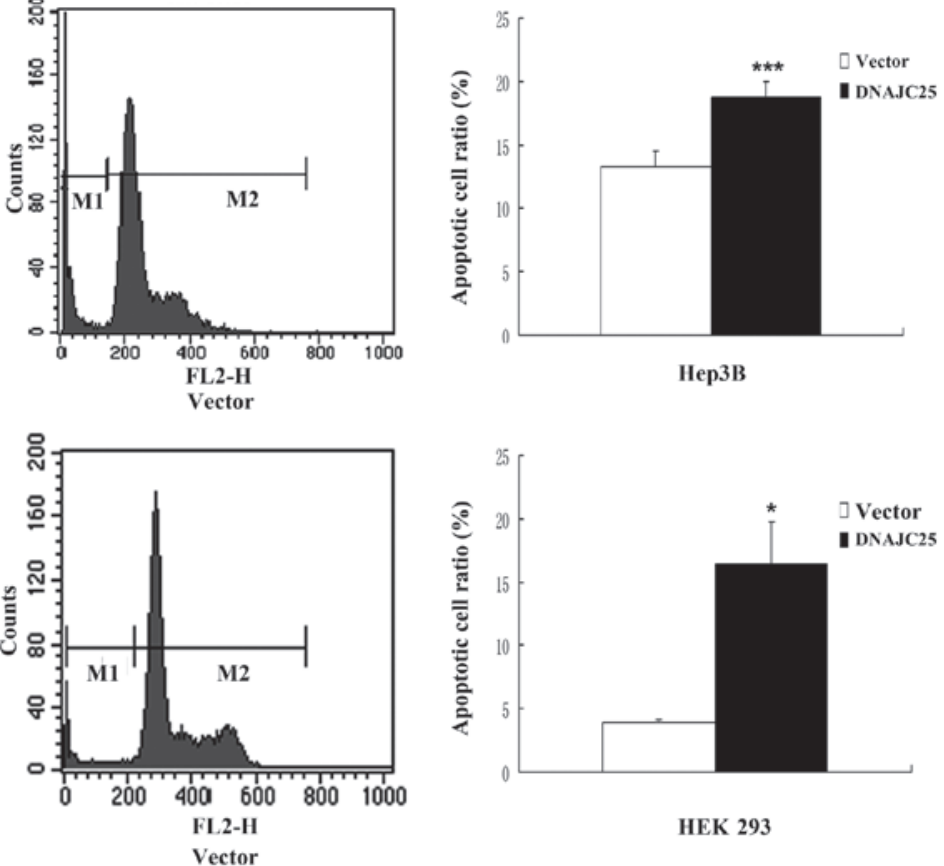

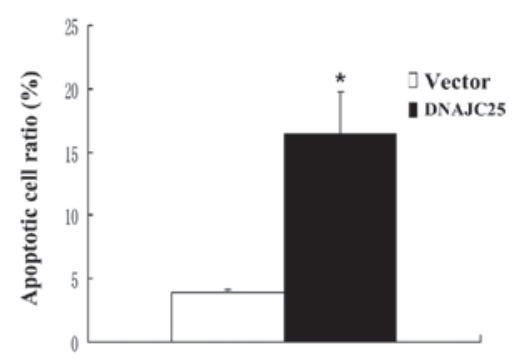

HEK 293

Figure 5. Overexpression of DNAJC25 promotes apoptosis. Hep3B and HEK 293 cells were transiently transfected with pCMV-Myc or pCMV-Myc-DNAJC25, harvested $36 \mathrm{~h}$ post-transfection and subjected to flow cytometric analysis. Sub-G1 phase cells were labeled as M1. Columns, mean (n=3); bars, SD. "P<0.05;

${ }^{* * * *} \mathrm{P}<0.001$ compared with control, t-test. Cell apoptosis was analyzed in (A) Hep3B and (B) HEK 293 cells.

\section{Discussion}

HSPs are best known as a group of proteins whose expression is increased when cells are exposed to stresses, such as high temperatures or oxygen deprivation (12). Further study revealed that HSPs are also found in cells under normal conditions $(13,14)$. HSPs are important in the metabolism of cells, immunological processes, cell cycle regulation, transcriptional activation and signal transduction (15-17). They act as 'chaperones', ensuring that their target proteins (client proteins) are 
in the right place and right shape at the right time. Therefore, HSPs aid the folding of proteins into the proper shapes and shuttle proteins from one compartment to another inside the cell, which is essential for their function $(18,19)$.

HSPs are broadly classified into six major families based on their relative molecular weights: HSP100, HSP90, HSP70, HSP60, HSP40 and small HSPs (such as HSP27) (20). Numerous HSPs are conserved throughout evolution, implying that their roles are important in the physiology of the cell.

To date, HSPs have been reported to be involved in the progression of a number of diseases. For instance, in autoimmune diseases, HSPs play indispensable roles in stimulating T-cell reactivity and protecting against disease (8). The upregulation of the synthesis of HSPs is considered to lead to tolerance of ischemia in certain animals (21). Additionally, HSPs are involved in the regulation of tumorigenesis. The overexpression of HSP27, 70 and 90 has been detected in various types of carcinoma, including $\operatorname{HCC}(9,22)$.

According to the domain similarity to the $E$. coli DnaJ protein, the HSP40 (DnaJ) family is further classified into three subclasses: DNAJA, DNAJB and DNAJC. In contrast to the well-studied HSP90 and HSP70 $(10,23,24)$, little is known concerning the role of HSP40 in tumor progression and metastasis. Only a few members of HSP40, including HLJ1 (DNAJB4), Tid1 (DNAJA3), MRJ (DNAJB6), JDP1 (DNAJC12) and HDJ2 (DNAJA1), have been determined to be associated with cancer in previous studies (25).

Previous studies have indicated that in canines, DNAJC25 is an ER-resident membrane protein. Its amino-terminal signal peptide comprised an ER-lumenal J-domain plus two transmembrane regions and another ER-lumenal domain (26). Our data showed that in HeLa cells and the HCC cell line SMMC-7721, the exogenous DNAJC25 fused with EGFP located in the cytoplasm.

To study the expression profile of this novel gene, we used RT-PCR to detect the distribution of DNAJC25 in 15 human tissues. The expression level of DNAJC25 in the liver was markedly higher than that in the other 14 tissues. In the thymus, prostate, testis, ovary, small intestine and colon, DNAJC25 was only expressed at trace levels. The markedly high expression of DNAJC25 in the liver led us to investigate the possibility of its correlation with the occurrence of diseases in the liver and study its function. The expression of DNAJC25 in HCC was further evaluated in 87 pairs of HCC specimens and adjacent normal liver tissues by quantitative real-time PCR. Our result indicated DNAJC25 was significantly downregulated in HCC, suggesting that DNAJC25 is involved in hepotocellular carcinogenesis and acts as a suppressor of HCC.

To explore the function of DNAJC25, we performed a colony formation assay on the HCC cell lines SMMC-7721 and Hep3B overexpressing DNAJC25. Our data indicate that the ectopic expression of DNAJC25 resulted in an inhibition of colony growth, which was consistent with the properties of a tumor suppressor to inhibit the ability of cells to initiate colonies and inhibit cell proliferation. Flow cytometry analysis further indicated that overexpression of DNAJC25 induced cell apoptosis in the HCC cell line Hep3B. A similar and also significant result was observed in HEK 293 cells, which may be attributed to the higher transfection efficiency. We also performed a cell cycle analysis, and no marked effect of DNAJC25 on the cell cycle was observed. This implies that the proapoptotic property may be the cause of the inhibition of cell growth by ectopic DNAJC25 expression.

In summary, we cloned and identified a new member of the DNAJC family, DNAJC25, explored its subcellular localization and tissue distribution and revealed its function as a candidate tumor suppressor in HCC for the first time. Notably, our description of both the downregulated expression of DNAJC25 in HCC and its proapoptotic function is opposite to the previous findings of certain other HSPs, such as HSP27 and HSP70, which have been reported to be upregulated in tumors and have antiapoptotic properties $(9,22)$. The present study has not only provided a new candidate suppressor of HCC, but also furthered the understanding of the HSP family. Further studies are required to validate its proapoptotic function and explore its potential role in cancer therapy.

\section{Acknowledgements}

This study was supported by the National Key Sci-Tech Special Project of China (grant no. 2008ZX10002-020).

\section{References}

1. Bosch FX, Ribes J, Diaz M and Cléries R: Primary liver cancer: worldwide incidence and trends. Gastroenterology 127 (Suppl 1): S5-S16, 2004.

2. Bosch FX, Ribes J, Cléries R and Díaz M: Epidemiology of hepatocellular carcinoma. Clin Liver Dis 9: 191-211, 2005.

3. Parkin DM, Bray F, Ferlay J and Pisani P: Global cancer statistics, 2002. CA Cancer J Clin 55: 74-108, 2005.

4. Lindquist S and Craig EA: The heat-shock proteins. Annu Rev Genet 22: 631-677, 1988.

5. Cotto JJ and Morimoto RI: Stress-induced activation of the heat-shock response: cell and molecular biology of heat-shock factors. Biochem Soc Symp 64: 105-118, 1999.

6. Schmitt E, Gehrmann M, Brunet M, Multhoff G and Garrido C: Intracellular and extracellular functions of heat shock proteins: repercussions in cancer therapy. J Leukoc Biol 81: 15-27, 2007.

7. Kopecek P, Altmannová K and Weigl E: Stress proteins: nomenclature, division and functions. Biomed Pap Med Fac Univ Palacky Olomouc Czech Repub 145: 39-47, 2001.

8. Raska M and Weigl E: Heat shock proteins in autoimmune diseases. Biomed Pap Med Fac Univ Palacky Olomouc Czech Repub 149: 243-249, 2005.

9. Khalil AA, Kabapy NF, Deraz SF and Smith C: Heat shock proteins in oncology: diagnostic biomarkers or therapeutic targets? Biochim Biophys Acta 1816: 89-104, 2011.

10. Soo ET, Yip GW, Lwin ZM, Kumar SD and Bay BH: Heat shock proteins as novel therapeutic targets in cancer. In Vivo 22: 311-315, 2008.

11. Ciocca DR and Calderwood SK: Heat shock proteins in cancer: diagnostic, prognostic, predictive, and treatment implications. Cell Stress Chaperones 10: 86-103, 2005.

12. Rylander MN, Feng Y, Bass J and Diller KR: Thermally induced injury and heat-shock protein expression in cells and tissues. Ann NY Acad Sci 1066: 222-242, 2005.

13. Schäfer C and Williams JA: Stress kinases and heat shock proteins in the pancreas: possible roles in normal function and disease. J Gastroenterol 35: 1-9, 2000.

14. Pirkkala L, Nykänen P and Sistonen L: Roles of the heat shock transcription factors in regulation of the heat shock response and beyond. FASEB J 15: 1118-1131, 2001.

15. Jäättelä M: Heat shock proteins as cellular lifeguards. Ann Med 31: 261-271, 1999.

16. Matijasevic Z, Snyder JE and Ludlum DB: Hypothermia causes a reversible, p53-mediated cell cycle arrest in cultured fibroblasts. Oncol Res 10: 605-610, 1998. 
17. Sonna LA, Fujita J, Gaffin SL and Lilly CM: Invited review: Effects of heat and cold stress on mammalian gene expression. J Appl Physiol 92: 1725-1742, 2002.

18. Hartl FU: Molecular chaperones in cellular protein folding. Nature 381: 571-579, 1996.

19. Lee $\mathrm{S}$ and Tsai FT: Molecular chaperones in protein quality control. J Biochem Mol Biol 38: 259-265, 2005.

20. Kampinga $\mathrm{HH}$ : Chaperones in preventing protein denaturation in living cells and protecting against cellular stress. Handb Exp Pharmacol 172: 1-42, 2006.

21. Snoeckx LH, Cornelussen RN, Van Nieuwenhoven FA, Reneman RS and Van Der Vusse GJ: Heat shock proteins and cardiovascular pathophysiology. Physiol Rev 81: 1461-1497, 2001.
22. Fields MM and Chevlen E: Ovarian cancer screening: a look at the evidence. Clin J Oncol Nurs 10: 77-81, 2006.

23. Bishop SC, Burlison JA and Blagg BS: Hsp90: a novel target for the disruption of multiple signaling cascades. Curr Cancer Drug Targets 7: 369-388, 2007.

24. Didelot C, Lanneau D, Brunet M, et al: Anti-cancer therapeutic approaches based on intracellular and extracellular heat shock proteins. Curr Med Chem 14: 2839-2847, 2007.

25. Mitra A, Shevde LA and Samant RS: Multi-faceted role of HSP40 in cancer. Clin Exp Metastasis 26: 559-567, 2009.

26. Zahedi RP, Völzing C, Schmitt A, et al: Analysis of the membrane proteome of canine pancreatic rough microsomes identifies a novel Hsp40, termed ERj7. Proteomics 9: 3463-3473, 2009. 